Reprod. Nutr. Dévelop., 1987, 27 (1 B), 171-188.

\title{
Adaptation de l'ingestion alimentaire aux dépenses énergétiques
}

\author{
Jeanine LOUIS-SYLVESTRE
}

\author{
Laboratoire de Neurobiologie de la Nutrition EPHE, \\ College de France \\ 11, place Marcelin Berthelot, 75231 Paris Cedex 05.
}

\section{Summary. Adaptation of food intake to expenditure.}

Body energy balance is regulated in adults. The accuracy of the phenomenon is particularly evident in laboratory animals under steady conditions. Moreover, it has been repeatedly demonstrated that this balance is maintained in spite of fluctuations in food intake or energy expenditure.

When animals such as rats, dogs or rabbits are presented with a diluted or concentrated version of familiar food, they compensate rapidly by increasing or decreasing their ponderal intake. This is achieved first by a change in meal frequency, then meal size adapts to the new caloric content and meal frequency returns to the original pattern. This adaptation is based on the learning of post-ingestive cues.

Hypo or hyperphagia leads to reduced or increased energy expenditure, as the case may be ; the basal metabolic rate is modulated by thyroid hormones and diet-induced thermogenesis by the sympathetic system. These variations are partly regulatory. In a cold environment, the increase in energy expenditure caused by increased thermogenesis is rapidly compensated by increased caloric intake.

Physical activity activates the sympathetic system responsible for numerous hormonal changes, the most important of which is insulin hyposecretion. In animals or humans, moderate aerobic exercice induces a small weight loss; afterwards, weight gain is normalized and increased caloric intake compensates for energy expenditures such as exercise, increased basal metabolic rate and diet-induced thermogenesis.

Extreme changes in body weight and fat are produced by gestation and lactation ; they are satisfactorily explained by concomitant hormonal changes. Especially during lactation, food intake is regulated so that it allows body weight to return to pregestation level.

Studies on the mechanisms implicated in the regulation of body energy balance are still in progress. Friedman and Ramirez (1985) suggest that the way fatty acids are utilized is important. Kasser et al. (1985) show a striking difference in the cellular metabolism of hypothalamic regions, depending on the metabolic state or the animal, and Woods et al. (1985) strongly suggest a role for the central insulin level. These hypotheses are welldocumented and not exclusive of each other.

\section{Introduction.}

Le bilan énergétique d'un animal adulte est équilibré. Apports et dépenses énergétiques sont tels que la masse énergétique représentée par les tissus maigres et les réserves adipeuses de l'organisme reste, en général, constante.

En conditions stables, chez l'animal de laboratoire, cet équilibre est particulièrement évident. Deux exemples précis en sont l'illustration : une étude portant sur 
10 rats mâles adultes $(358 \pm 4 \mathrm{~g}$ ) a montré qu'ils ingéraient $20,9 \pm 0,2 \mathrm{~g}$ par jour. La valeur de cette erreur standard est remarquable, d'autant plus qu'elle est parfaitement expliquée par une faible mais constante prise de poids $(0,7 \mathrm{~g}$ par jour) au cours des 20 jours considérés (Le Magnen et Devos 1980). En effet chez quelques espèces, il a été constaté que les adultes présentent une adiposité faiblement, mais constamment, croissante ; ce pourrait être le fait d'une trajectoire préprogrammée, mais il semble que la grande sédentarité en soit généralement la cause. Huit singes adultes pesant de 5,7 à $8,8 \mathrm{~kg}$ furent étudiés (Hansen et al., 1981) chacun sur des périodes de 15 à 60 jours. Leur prise calorique moyenne était de $621 \pm 3,3 \mathrm{kcal}$ soit $84 \pm 0,7$ par $\mathrm{kg}$ de poids corporel. La prise de poids concomitante fut de 0 à $0,24 \mathrm{~kg}$ par semaine.

La démonstration simple d'une régulation précise de l'adiposité est faite par deux sortes de manipulations : sous-poids ou sur-poids obtenus par gavage sont rapidement corrigés, à la cessation du traitement, par l'adaptation spontanée de la prise alimentaire; l'ablation ou l'addition chirurgicale de masses adipeuses entraîne aussi une correction par la variation spontanée de l'entrée d'énergie.

Cette courte revue fera le point actuel des connaissances concernant le maintien chez l'animal adulte de l'équilibre du bilan énergétique en dépit de fluctuations importantes dans les apports énergétiques et/ou dans la disponibilité des aliments, et en dépit aussi, de fluctuations dans les dépenses provoquées par l'exercice physique plus ou moins intense, l'exposition à des températures environnantes variables et des états physiologiques remarquables tels gestation et lactation.

La grande majorité des travaux dans ces domaines a été effectuée sur le rat. Afin de dresser un tableau cohérent, les résultats évoqués seront ceux qui ont été acquis sur cet omnivore. Beaucoup de ces données ont pu d'ailleurs être confirmées par des études parallèles effectuées sur sujets humains.

\section{Fluctuation des apports alimentaires.}

L'ensemble des résultats expérimentaux montre à l'évidence que l'équilibre du bilan énergétique est obtenu principalement par le contrôle des entrées d'énergie. La régulation par le' contrôle des dépenses est en général un adjuvant; à elle seule, elle ne peut qu'être imparfaite.

\section{- Le phénomène de l'adaptation calorique.}

L'adaptation du volume ingéré à la densité calorique de l'aliment est un phénomène bien connu, exploré depuis les travaux de Adolph sur le rat en 1947. C'est une démonstration éclatante de l'efficacité du contrôle des entrées.

Mises à part lẹs expériences dans lesquelles la dilution calorique est telle que le volume à ingérer dépasse, soit les possibilités du tractus digestif, soit le temps dévolu à l'ingestion, le phénomène de l'adaptation calorique a été retrouvé chez la majorité des espèces animales : chien, rat, ruminants, porc, poulet, homme, etc. Des modifications importantes de palatabilité ont quelques fois pu le masquer: c'est ainsi que la dilution de l'aliment habituel avec du kaolin ne convient pas au chat qui, cependant, régule parfaitement son ingestion si la dilution est faite avec de l'eau (Castonguay, 1981). 
Comment s'opère cet ajustement? Un travail déjà ancien de Booth (1972 a, b) montre que, chez le rat, la dilution de son aliment complet par la cellulose étant de $50 \%$, au bout de trois jours le volume quotidien ingéré est très supérieur au volume de base ; en 16 jours l'ajustement est parfait. L'enregistrement de la séquence alimentaire a montré que le phénomène débute par une augmentation de la fréquence des repas ; leur dimension croît ensuite, peu à peu (l'aliment étant dilué de $50 \%$, le premier repas dont le volume est significativement plus grand, est au plus tôt le quatrième et généralement le sixième) (Snowdon, 1969). Ces observations entraînent plusieurs remarques. Il a été longuement démontré pour de nombreuses espèces que, à l'état stable, la quantité de calories ingérée au cours d'un repas et la durée de l'intervalle de non consommation qui suit, sont fortement corrélées. L'augmentation de fréquence des repas isovolumiques, mais hypocaloriques, que fait l'animal soudainement soumis à un régime dilué, confirme cette corrélation. Le retour à la fréquence habituellement constatée par augmentation progressive du volume ingéré à chaque repas ressortit de deux phénomènes : on peut penser, à la suite de Collier (1985) qu'il répond à la loi très générale du meilleur rendement global ou de la meilleure économie (rapport entre le travail nécessité par l'obtention de la nourriture, la digestion, l'absorption, le métabolisme et l'apport calorique résultant) pour l'animal. Cette fréquence optimale étant recherchée par l'animal, c'est par un apprentissage lié aux phénomènes post-ingestifs que l'animal apprend inconsciemment à ingérer de volumineux repas quand l'aliment est caloriquement dilué. Booth (1972 a, b) a remarquablement démontré comment l'animal " apprend " la teneur en calories des aliments quand une reconnaissance sensorielle lodeurs différentes selon la dilution par exemple) est possible.

Des expériences très récentes (Treit et Spetch, 1986) semblent montrer que chez le rat une appréciation immédiate du contenu calorique d'un aliment nouveau soit possible. Les animaux sont mis et maintenus à $90 \%$ de leur poids normal ; ils reçoivent chaque jour pendant 7 jours " un petit repas " et 4 heures après le reste de la ration calculée. Le jour du test, le " petit repas » est ad libitum. II est constitué d'une solution à 20 ou $50 \%$ d'huile dans l'eau. Les quantités ingérées, mesurées, varient en sens inverse de la concentration. Ce résultat surprenant vient cependant à l'appui d'études faites chez l'homme (Campbell et al., 1971) et qui suggèrent que, dans certaines limites, une mesure directe et instantanée peut intervenir quand l'apport calorique et donc l'information ont été limités. Le contrôle par apprentissage interviendrait dans le cas général d'un accès illimité à l'aliment.

\section{- Prise alimentaire excédentaire ou réduite.}

Quand la prise alimentaire est accrue ou restreinte, les dépenses respectivement augmentent ou diminuent. Cette régulation, imparfaite cependant, a été d'abord suggérée par les travaux sur le sujet humain puis, en 1979, par une étude sur le rat (Rothwell et Stock 1979 a, b). Des rats adultes (3-9 mois) mis sur un régime composé d'aliments variés et palatables (régime cafétéria) ingèrent spontanément $34 \%$ de plus d'énergie que les rats témoins, mais dépensent $25 \%$ de plus d'énergie (Rothwell et Stock, 1983). 
Après ingestion 'd'un repas, la dépense énergétique augmente transitoirement (pendant environ $4 \mathrm{~h}$ chez l'homme). Cette "thermogénèse postprandiale " ou cet « effet thermique de la nourriture » a été longtemps considéré comme le coût énergétique obligatoire de l'ingestion, digestion, absorption, utilisation immédiate ou différée des nutriments. Sa mesure a souvent mais pas toujours, montré qu'elle pourrait être régulatrice. Dans le cas d'une prise énergétique chroniquement excédentaire la mesure de la dépense énergétique concomitante, réalisée sur des périodes allant de $24 \mathrm{~h}$ à 7 jours, a permis, le plus souvent, de mettre en évidence le rôle partiellement régulateur de la dépense. Cette thermogénèse excédentaire régulatrice comprend la thermogénèse post-prandiale dans sa part régulatrice, mais relève aussi d'un autre poste dans les dépenses puisqu'elle déborde de plusieurs jours la durée de la prise excédentaire (revue de Louis-Sylvestre, 1984).

La restriction calorique entraîne une réduction du métabolisme de repos, mais aussi une épargne sur le coût de la mise en réserve, une épargne sur l'activité physique et sur la thermogénèse facultative induite par le régime. Chez le rat, une étude très complète des effets de la restriction à long terme sur les différents postes de l'utilisation de l'énergie ingérée vient d'être publiée (Hill et al., 1985). Elle montre que l'épargne est proportionnelle à la réduction de poids corporel, mais atteint un maximum qui ne peut être dépassé, même par une restriction plus sévère ; à poids constant, l'épargne s'élève avec le temps ; elle est principalement due à une baisse du métabolisme de repos. Plusieurs auteurs (Boyle et al., 1978, Cumming et Morrison, 1960) ont montré que la réduction du métabolisme persistait quelque temps pendant la période de réalimentation, expliquant ainsi une prise de poids galopante à la cessation de la restriction.

Les données concernant les possibles mécanismes de cette épargne viennent d'une étude récente (Rothwell et al., 1985) portant sur des rats mis à jeun pendant 3 jours et nourris à nouveau. Le jeûne entraîne une chute de 20 à $25 \%$ de la consommation d'oxygène qui ne peut être expliquée par la seule chute de poids. A cet hypométabolisme est associée une réduction des taux circulants d'hormones thyroïdiennes qui n'en est cependant pas directement responsable puisque l'injection de triiodothyronine T3 ne rétablit pas la consommation d'oxygène. Dans ces conditions, la prise d'une petite quantité de glucides $(40 \mathrm{KJ})$ ou de protides provoque, $24 \mathrm{~h}$ après, ùne élévation de consommation d'oxygène qui est supprimée par le propranolol ( $\beta$-antagoniste). Il semble donc qu'une prise alimentaire insulinogénique provoque une activation du système nerveux sympathique qui serait à l'origine d'une élévation de la T3 plasmatique, expliquant ainsi le retour à la normale du métabolisme. L'ensemble de l'épargne constatée lors de la restriction ou du jeûne total semble donc être lié à la réduction de l'activation sympathique. Deux sortes de travaux confirment cette hypothèse. Les premiers concernent la modulation de l'activité sympathique par la quantité et la composition des ingesta (Young et Landsberg, 1977 a, b). Les seconds font appel aux similitudes entre thermogénèse induite par le froid et thermogénèse induite par le régime ; portant en particulier sur le rongeur, ces travaux soulignent eux aussi le rôle du système sympathique mais s'intéressent tout particulièrement à son impact sur le tissu adipeux brun (Rothwell et Stock, 1979 a, b).

Les relations entre niveau de prise alimentaire et activité sympathique ont fait 
l'objet d'une revue récente (Landsberg et Young, 1981). Chez I'animal et l'homme, le jeûne, la restriction calorique diminuent l'activité sympathique. Chez le rat il s'agit d'une réduction du taux de renouvellement de la noradrénaline (NA) au niveau de nombreux organes. Chez l'homme, on a constaté une réduction du taux de NA circulant sur le sujet obèse en restriction et chez les malades atteints d'anorexie mentale ; chez le sujet normal, 3 jours de jeûne entraînent une excrétion urinaire réduite de NA. Chez I'homme également, la prise alimentaire, en particulier gludidique, accroît le taux de NA plasmatique, sans cependant affecter le taux d'adrénaline ; le taux de renouvellement de NA est augmenté aussi bien chez I'homme que chez le rat et ceci après une suralimentation, même modeste, mais en particulier glucidique.

Le rôle majeur de la prise glucidique et des ses conséquences sur la sécrétion d'insuline dans cette activation sympathique est largement confirmé par des expériences de perfusions qui imposent au sujet des niveaux constants de glucose et/ou d'insuline (Rowe et Young, 1981). Beaucoup font l'hypothèse selon laquelle l'insulinémie, dont le niveau semble moduler l'activité sympathique, pourrait être le signal qui, au niveau du SNC, couplerait activité sympathique, donc thermogénèse, et prise alimentaire. Les résultats obtenus sur animaux (souris-rat) lésés au niveau de I'hypothalamus ventro-médian suggèrent que ce signal agirait sur le métabolisme insulino-dépendant de cette zone (Perkins et al., 1981). Ce rôle de l'insuline est corroboré par quelques points remarquables : chez l'obèse la thermogénèse régulatrice est d'autant plus défectueuse que l'insulinorésistance est plus grande (Ravussin et al., 1985). Cette thermogénèse dépend du régime et de son pouvoir insulinogénique et évolue en fonction de la durée de I'hyperconsommation (Levin et al., 1983). Cependant un certain nombre de résultats, quelquefois très récents, obtenus chez l'homme, apportent des faits contradictoires. Pour les uns, il n'y a pas de thermogénèse régulatrice induite par le régime (Van Es et al., 1982) ; pour les autres, celle-ci ne fait pas intervenir le système sympathique.

Chez le rongeur, l'importance du tissu adipeux brun et de son innervation sympathique ne fait plus de doute (Stock et Rothwell, 1985). Une thermogénèse hépatique (Rothwell et Stock, 1984) ou musculaire (Astrup et al. 1986) n'est pas exclue.

De même doit être envisagé le rôle des hormones thyroidiennes. La restriction calorique entraîne une réduction de la concentration plasmatique en triiodothyroine (T3) et une augmentation de rT3 consécutive à la réduction de la conversion périphérique de T4 en T3 et aussi à celle de la clairance métabolique de rT3. La surconsommation entraîne les changements inverses (Stockholm et Hansen, 1983, Bogardus et al., 1982, Danforth et al., 1979, Burger et al., 1980). II semble que le taux de T3 libre (Danforth, 1983) ainsi que la liaison de T3 à ses sites récepteurs (Schlusser et Orlando, 1978) soient directement corrélés à la prise calorique ; la relation de cause à effet reste à établir.

Ainsi les manipulations nutritionnelles modulent l'activité sympathique et, simultanément, taux et activité des hormones thyroidiennes; il semble que les deux mécanismes soient liés. 


\section{Fluctuation des dépenses}

\section{- Exercice physique.}

Le bilan énergétique de l'animal ou du sujet humain soumis à l'exercice physique a fait l'objet de très nombreux travaux. Le type d'exercice choisi, son intensité, la durée quotidienne, la durée de l'entraînement sont tellement différents que les résultats sont souvent difficilement interprétables.

Les plus intéressants concernent l'exercice physique, léger ou modéré, toujours aérobie. Aussi, chez le rat, les travaux qui seront évoqués portent sur des exercices de marche sur tapis roulant (par exemple $1 \mathrm{~km} / \mathrm{h}$ pendant une heure) ou des exercices de nage ( $2 \mathrm{~h}$ par jour) pratiqués quotidiennement pendant 1 ou 2 semaines et jusqu'à 10 semaines. Ces exercices correspondent en moyenne pour un rat de $250 \mathrm{~g}$ à une dépense calorique brute de $5 \mathrm{kcal} / \mathrm{h}$ soit $200 \%$ de la valeur de base.

Chez un homme, il s'agit de marches de $2 \mathrm{~h}$ environ sur tapis roulant, à la vitesse de 4 à $6 \mathrm{~km} / \mathrm{h}$, nécessitant une consommation moyenne de 1 à 2 litres d'oxygène $/ \mathrm{mn}$, correspondant à une dépense brute de 380 à $780 \mathrm{kcal} / \mathrm{j}$; c'est-àdire que la dépense quotidienne totale atteint 110 à $125 \%$ de la valeur de base. Selon les études, ces exercices sont pratiqués pendant quelques jours ou quelques semaines.

Dans ces conditions, chez le rat, prise alimentaire et poids corporel diminuent au début de l'entraînement (Nance et al., 1977, Katch et al., 1979, Gleeson et al., 1982) puis la prise alimentaire augmente. Gleeson et al. montrent qu'après 3 semaines d'entraînement la prise alimentaire des rats traités n'est pas différente de celle des rats demeurés sédentaires. Après 5 semaines, les premiers consomment $25 \%$ de plus d'aliments par $100 \mathrm{~g}$ de poids que les témoins. Le poids corporel des rats à l'exercice est alors équilibré à un niveau inférieur de $60 \mathrm{~g}$ à celui des sédentaires. Confirmant en cela de nombreux résultats obtenus chez l'animal et le sujet humain, Gleeson et al. montrent aussi que l'exercice augmente d'environ $7 \%$ le métabolisme de repos et que la thermogénèse induite par l'ingestion est multipliée par 1,6 (Miller et Munford, 1967 : Gleeson et al., 1979 ; Segal et Gutin 1983 ; Segal et al., 1985 - Leblanc et al., 1985).

Les résultats obtenus sur des rats femelles à l'exercice sont différents : en général, la prise alimentaire est augmentée dès le début de l'exercice et le poids est maintenu (Nance et al., 1977 ; Richard et Leblanc, 1980). Ce phénomène est mal expliqué.

Quelques études soigneuses (Warnold et Lenner, 1977 ; Woo et Pi-Sunyer, 1985) réalisées sur le sujet humain de poids normal montrent que, dès le début de l'entraînement et durablement, la balance énergétique est maintenue. La prise alimentaire semble d'emblée adaptée à l'augmentation des dépenses. La compensation est réalisée par une augmentation principale de la prise de nutriments glucidiques.

Les connaissances concernant la réponse hormonale à l'exercice sont encore fragmentaires. Certains changements hormonaux ont lieu tôt au cours de l'exercice et ne sont certainement pas la réponse à des stimuli métaboliques. Galbo 
(1983) fait l'hypothèse selon laquelle, au début de l'exercice, des influx venant des muscles au travail et des centres moteurs moduleraient l'activité de centres nerveux au niveau central en fonction du travail effectué. Ces centres, en retour, induiraient une augmentation de sécrétion de certaines hormones hypohysaires et aussi une augmentation de l'activité sympatho-surrénalienne.

Au cours de l'exercice, l'activité du système sympatho-surrénalien et donc les concentrations plasmatiques en catécholamines, noradrénaline, adrénaline et dopamine augmentent chez tous les vertébrés supérieurs. Chez l'homme, par exemple, on a pu montrer que l'augmentation en catécholamines est directement liée à la consommation d'oxygène (Lewis et al., 1983).

Cette augmentation a une importance majeure en ce qui concerne l'adaptation cardiovasculaire et la thermorégulation, les pertes en eau et en électrolytes. Les conséquences hormonales de cette activation sympathique sont indiquées sur la figure 1. L'effet le plus important est sans doute l'inhibition $\alpha$-adrénergique de la sécrétion d'insuline; chez l'homme, cette inhibition est exercée par les nerfs sympathiques; chez le rat, l'adrénaline est concernée.

\section{EXERCICE}

Influx venant des $\left\{\begin{array}{l}\text { muscles } \\ \text { centres moteurs }\end{array} \longrightarrow\right.$ Système nerveux central

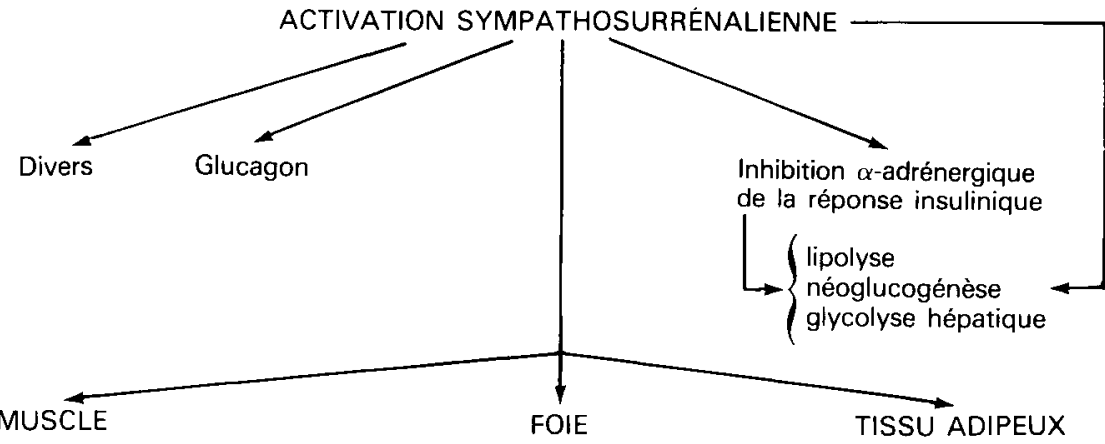

$$
1
$$

sensibilité à l'insuline hexokinase

glycogène synthétase $\downarrow$

La captation du glucose reste optimale

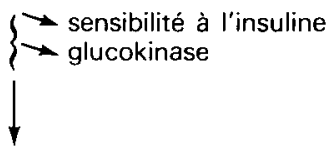

Réduction de la captation du glucose

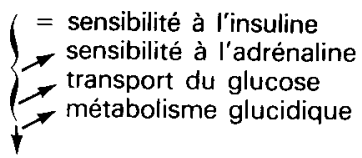

Le renouvellement des triglycérides est grandement augmenté

FIG. 1.

A la suite de l'exercice, la normalisation de l'homéostasie et des niveaux hormonaux est rapide si l'exercice n'est ni prolongé ni intense. C'est ainsi que l'insulinémie peut présenter un rebond (Galbo et Gollnick, 1984) dû à la cessation de l'inhibition adrénergique. Si l'exercice est prolongé, l'insulinémie peut rester basse 
longtemps et être accompagnée d'une hyposensibilité des cellules $\beta$ au glucose (James et al. 1983). L'entraînement apporte des changements durables dont une hyposensibilité des cellules $\beta$ au glucose. Cet effet bénéfique de l'exercice sur la demande insulinique est important : toutes choses égales d'ailleurs, la sécrétion insulinique totale au cours du nycthémère est, chez les sujets entraînés, inférieure de $40 \%$ à celle des sujets sédentaires. Ce bénéfice est rapidement acquis (après une heure d'exercice intense chez un sujet habituellement sédentaire) mais s'évanouit rapidement (il n'en reste rien après trois jours de repos chez des sujets entraînés) (Leblanc et al., 1983, 1981).

Cet hypoinsulinisme facilite la lipolyse, la néoglucogénèse, la glycogénolyse hépatique. II pourrait empêcher l'utilisation du glucose par le muscle ; mais on a récemment montré à ce niveau une augmentation importante de sensibilité à l'insuline $(17 \%)$. Cette sensibilité accrue, accompagnée d'une activation enzymatique (hexokinase et glycogène-synthétase) explique pourquoi la captation musculaire de glucose reste optimale malgré l'hypoinsulinisme général et explique aussi comment est favorisé le stockage du glycogène par le muscle. Cette augmentation de sensibilité n'est produite qu'au niveau musculaire ; la sensibilité reste inchangée en ce qui concerne le tissu adipeux et est réduite au niveau hépatique (Mondon et al., 1980). Dans le foie, le phénomène entraîne une diminution de l'activité glucokinasique et donc une réduction de la captation du glucose (Zawalich et al., 1982).

Au niveau du tissu adipeux, une augmentation ( 2 à 3 fois) de la capacité à métaboliser le glucose a été mise en évidence. Ceci n'est dû, ni à un changement de sensibilité à l'insuline, ni à un changement de liaison de l'insuline, mais reflète une activation du métabolisme glucidique intracellulaire. De plus, la sensibilité à l'adrénaline est multipliée par 3 à 4 . Ainsi, l'entraînement augmente le transport du glucose et son métabolisme intracellulaire vers la lipogénèse et sous l'effet de l'adrénaline, la lipolyse augmente aussi. Donc, l'entraînement augmente la capacité de renouvellement des triglycérides du tissu adipeux (Wardzala et al., 1982 ; Vinten et al., 1985).

Les caractéristiques essentielles des voies métaboliques de l'exercice sont résumées sur la figure 1 . Quelques remarques générales peuvent éclairer la situation. La réponse hormonale à l'exercice n'est pas discriminante et provoque la mobilisation du glycogène et des triglycérides des réserves intra- et extramusculaires. La quantité de carburants mobilisés depuis les différentes réserves dépend de la réponse hormonale, du niveau d'activité physique, de la dimension des réserves, de l'état des récepteurs hormonaux et de l'activité des enzymes concernés. Les changements hormonaux induits, la sensibilité des différentes réserves à la stimulation, la capacité du muscle à métaboliser les différents substrats dépendent, eux, de l'état de nutrition, du niveau d'activité physique et du niveau d'entraînement du sujet. La modulation de la prise alimentaire en fonction de l'exercice et du niveau d'entraînement répond à ces cascades d'événements.

\section{- Exposition au froid.}

Le phénomène brut est très clair et retrouvé dans toutes les espèces considérées : le maintien de longue durée en ambiance froide provoque une baisse de 
poids corporel et une augmentation de prise calorique ramenée au poids. Chez le rat maintenu a $5{ }^{\circ} \mathrm{C}$, cette augmentation de prise alimentaire s'installe en quelques jours. Pendant les deux premiers jours de l'exposition, la fréquence des repas nocturnes diminue alors que leur taille ne varie pas; fréquence et taille des repas diurnes ne sont pas affectés. Au bout d'une semaine, les caractéristiques définitives de la séquence alimentaire sont acquises : la fréquence des repas nocturnes est basse, leur dimension est augmentée de $200 \%$; de jour, la fréquence est inchangée, la dimension augmentée de $141 \%$ (Leung et Horwitz, 1976). Ainsi au froid, malgré une augmentation des dépenses, I'hyperphagie compensatrice apparaît lentement, l'animal commençant par utiliser ses réserves; la balance énergétique, négative d'abord, s'équilibre en une semaine et le sous-poids obtenu est ensuite maintenu. Au retour à la température ambiante normale, le retour à la séquence et à la quantité ingérée préalables est immédiat ; l'animal reprend très vite le poids des animaux non exposés au froid.

Il est intéressant de constater que, lorsque l'animal (rat ou souris) peut choisir ses nutriments, une augmentation de prise calorique se fait uniquement sur les glucides (Leshner et al., 1971 ; Donhoffer et Vonotsky, 1947). La même sélection est réalisée lorsqu'une injection de thyroxine est pratiquée sur des animaux laissés à $22{ }^{\circ} \mathrm{C}$. La fréquence des repas et la prise calorique augmentent immédiatement lorsque les animaux, préalablement à jeun ( $24 \mathrm{~h})$, sont maintenus au froid sur aliment liquide $(6 \mathrm{~h})$. Dans ces conditions, interviennent une augmentation de vitesse de vidange gastrique, mais aussi le stimulus froid per se et ceci, seulement pendant la période d'ingestion ; en effet si l'animal subit le jeûne au froid mais est alimenté à $22{ }^{\circ} \mathrm{C}$, aucune augmentation de prise calorique n'est observée (Kraly et Blass, 1976). Chez l'homme, la comparaison des dépenses énergétiques faites en $30 \mathrm{~h}$, soit à $28^{\circ} \mathrm{C}$ soit à $22{ }^{\circ} \mathrm{C}$, montre qu'une baisse de $6^{\circ} \mathrm{C}$ dans la température ambiante ne provoque pas de frissons, mais entraîne une augmentation de 7 \% dans la production de chaleur. Cette étude montre aussi que la thermogénèse induite par la prise des repas se subtitue partiellement à la thermogénèse induite par le froid. II est intéressant de calculer que, toutes choses étant égales par ailleurs, si un sujet humain passe $10 \%$ de son temps à $22^{\circ} \mathrm{C}$, il dépense l'équivalent de $1 \mathrm{~kg}$ de réserves adipeuses en un an (Dauncey, 1981).

Les études classiques de Hsieh et al. (1957), réalisées il y a trente ans maintenant, ont bien démontré le rôle du système nerveux syspathique dans la régulation de la thermogénèse au froid. Cette activation accroît la conservation de chaleur en provoquant une vasoconstriction périphérique et la piloérection. Elle augmente aussi la production de chaleur en facilitant le frisson et surtout en stimulant la production de chaleur métabolique par le tissu adipeux brun (TAB), le foie ou encore les muscles squelettiques.

La stimulation sympathique est déclenchée par l'activité de neurones sensibles au froid situés à la périphérie (peau) ou au niveau central (hypothalamus, tronc cérébral, moelle épinière). Ces influx sont intégrés au niveau hypothalamique avec pour résultat l'activation sympathique. Le système sympatho-surrénalien joue un rôle majeur dans la mobilisation des carburants de réserve (Landsberg et al., 1984). Mobilisation des triglycérides du tissu adipeux, du glycogène dans le foie et le muscle, synthèse du glucose et des corps cétoniques au niveau du foie 
sont en effet impliqués. Chez les animaux maintenus en ambiance froide et soumis à une médullectomie surrénalienne et à une sympathectomie chimique, la lipolyse et la sortie de glucose hépatique ne sont plus augmentées (Landsberg et al., 1984). II semble que ce soit l'innervation sympathique directe du tissu adipeux qui joue le rôle majeur dans la stimulation de la lipolyse (fig. 2). La NA sécrétée activerait la lipase hormonosensible; cet effet serait médié par les récepteurs $\beta$ adrénergiques et serait dépendant du système adényl-cyclase-AMP cyclique. Ainsi, les réserves corporelles adipeuses fournissent une part importante de l'énergie requise. Ce déficit est ensuite très vite en partie compensé par l'augmentation de prise alimentaire entraînée par ces changements du métabolisme. Il faut cependant remarquer qu'une légère déplétion des réserves grasses demeure même quand l'animal dispose de nourriture ad libitum (O'Hara et al., 1979).

L'exposition au froid touche aussi le métabolisme glucidique; les réserves glycogéniques ne sont pas affectées et l'utilisation glucidique est augmentée.

FROID

Neurones sensibles $\longrightarrow$ activation sympathique

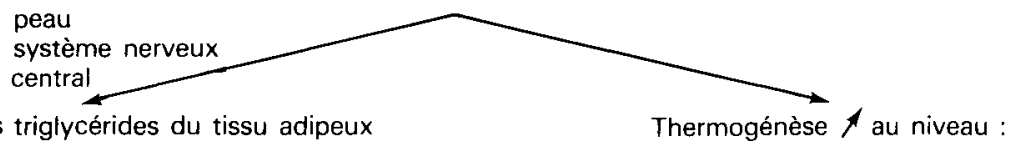

Mobilisation des triglycérides du tissu adipeux

Mobilisation du glycogène du foie du muscle

Synthèse du glucose dans le foie des corps cétoniques

$\begin{array}{ll}\text { Vasoconstriction } & \text { Tissu adipeux } \\ \text { Piloérection } & \text { brun } \\ & \text { Foie } \\ & \text { Muscle }\end{array}$

FIG. 2.

\section{- Gestation-Lactation.}

Gestation et lactation sont des exemples d'états physiologiques normaux au cours desquels se produisent des variations importantes de poids et de composition corporelle; les réserves adipeuses maternelles augmentent durant la gestation : leur mobilisation et l'hyperphagie rendent compte de la demande énergétique due à la production de lait. A la lumière de travaux récents effectués chez le rat, il semble qu'un rôle majeur puisse être attribué aux hormones ovariennes.

Les stéroïdes ovariens et testiculaires ont des effets importants sur la prise alimentaire, le poids et la composition corporelle. Très brièvement, les faits sont les suivants : l'ovariectomie induit l'hyperphagie et une augmentation importante de la masse adipeuse ; le traitement à l'œstradiol renverse le phénomène ; la progestérone seule n'a pas d'effet sur la prise alimentaire et la composition corporelle, mais en présence d'œstradiol, elle induit hyperphagie et adiposité. Les fluctuations de la prise alimentaire et du poids corporel constatées durant le cycle œstrien, la gestation et la pseudogestation sont en parfait accord avec les variations hormonales impliquées et leurs effets expérimentalement établis.

La cascade des événements physiologiques qui lie prise alimentaire, état des réserves et hormones sexuelles est loin d'être claire. Dans une excellente publica- 
tion, Wade et Gray, (1979) passent en revue les différentes possibilités ; I'hypothèse la plus séduisante fait appel au jeu des stéroïdes sur le métabolisme lipidique. L'œstradiol élève le taux des triglycérides sanguins en réduisant l'activité lipoprotéine-lipase au milieu du tissu adipeux et du foie et en stimulant la synthèse hépatique des triglycérides. La progestérone, en présence d'œestradiol, diminue les triglycérides sériques en stimulant à la fois l'activité lipoprotéine-lipase au niveau mammaire et adipeux et l'activité triglycéride-lipase au niveau hépatique. Ce sont ces effets sur les tissus cibles (tissu adipeux, foie en particulier) qui, entraînant des variations dans les métabolites (triglycérides) circulants, induiraient les changements de prise alimentaire constatés. Le travail de Young et al. (1978), montrant que I'hyperphagie induite par le traitement à l'cestradiol est plus importante si le régime proposé est très lipidique, renforce l'hypothèse proposée (fig. 3 ).

\section{GESTATION}

Hormones ovariennes et métabolisme lipidique

$$
\begin{aligned}
& \text { œestradiol }\left\{\begin{array}{l}
\text { triglycérides sériques } \\
\text { lipoprotéine-lipase du tissu adipeux et du foie } \\
\text { triglycéride-lipase du foie }
\end{array}\right. \\
& \underset{+}{\text { progestérone }} \begin{array}{c}
\text { triglycérides sériques } \\
\text { cestradiol }
\end{array}\left\{\begin{array}{l}
\text { lipoprotéine-lipase du tissu adipeux et du foie } \\
\text { triglycéride-lipase du foie }
\end{array}\right.
\end{aligned}
$$

Hormones ovariennes et modulation de l'activité physique par effet direct sur l'aire préoptique médiane

FIG. 3.

Au cours de la lactation, la prolactine pourrait être responsable de la suppression de l'activité lipoprotéine-lipase du tissu adipeux, phénomène qui aiderait à la fourniture d'acides gras à longue chaîne pour la glande mammaire (fig. 4).

\section{LACTATION}

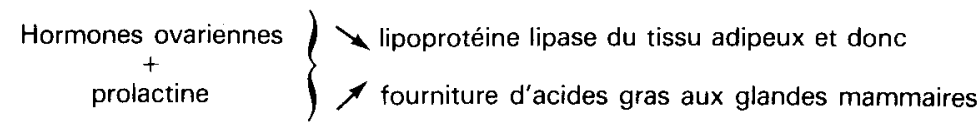

Suppression de l'activité thermogénique du tissu adipeux brun

FIG. 4.

Quelques points ayant fait l'objet de recherches récentes méritent d'être soulevés. Chez la ratte, pendant la gestation, l'augmentation de prise alimentaire est induite par l'augmentation de progestérone, la prise de poids corporel maternelle est indépendante du poids de départ; prise alimentaire et prise de poids sont cependant dépendantes du régime. Ainsi, femelles de poids normal et femelles obèses mises sur le même régime ont le même gain de poids (Rolls et Rowe, $1982)$; le régime cafétéria est efficace, de la même façon, sur les deux groupes 
(Rolls et al., 1984). Le phénomène est très différent durant la lactation : il y a une forte corrélation positive entre le poids à la parturition et la perte de poids durant la lactation. La palatabilité du régime intervient encore (Rolls et al., 1984). II semble donc que le gain de poids ne soit pas régulé pendant la gestation ; au cours de la lactation il y a normalisation du poids, la perte régulatrice de poids dépendant du poids au moment de la parturition.

Les dépenses énergétiques répondent aussi aux hormones sexuelles : l'augmentation d'activité physique suit la sécrétion d'œestradiol durant le proestrus et l'augmentation de progestérone est accompagnée d'une baisse de cette activité (Wade et Gray, 1979). II semble que l'œstradiol agisse directement sur l'aire préoptique médiane pour stimuler l'activité physique (Wade et Zucker, 1970). Ainsi, chez le rat, femelies en gestation et en lactation sont peu actives ; simultanément la thermogénèse du tissu adipeux brun (TAB) est fortement ralentie. Trayhurn (1985) montre que la liaison guanosine diphosphopyridine (GDP), témoin de l'activité du TAB, est fortement diminuée par la gestation et la lactation. Le poids corporel et le régime sont sans effet sur la thermogénèse des femelles en lactation. Il est vraisemblable que l'augmentation importante de production de chaleur due au métabolisme oblitère chez ces animaux le besoin de chaleur thermorégulatrice. L'importance de la zone de neutralité thermique constatée confirme le phénomène. Celui-ci est important : l'établissement du bilan chez la souris montre que la perte d'énergie corporelle maternelle durant la lactation est de $3,7 \mathrm{~kJ}$ par jour ; la diminution de thermogénèse est de $25 \mathrm{~kJ}$ par jour.

Le choix alimentaire au cours des différents états physiologiques précédemment évoqués a fait l'objet de quelques travaux qui le décrivent, constatent qu'il est adapté, mais n'en expliquent pas le mécanisme. Chez le rat et le sujet humain, au cours du cycle sexuel, les variations de prise alimentaire touchent uniquement les glucides (Wurtman et Baum, 1980, Dalvit-Mc Phillips, 1983). De plus, pendant la phase lutéale, une forte inclination vers les aliments sucrés a été remarquée (Morton et al., 1953). En cas de choix possible au cours de la gestation et de la lactation, l'augmentation de prise alimentaire est réalisée par l'augmentation de la prise protéique (Leshner, 1972). Ceci confirme absolument d'autres études montrant que la sélection spontanée opérée répond aux besoins de l'organisme.

\section{Mécanismes de régulation. - Hypothèses actuelles.}

Les mécanismes généraux impliqués dans cette régulation ont fait l'objet d'hypothèses qui prennent appui sur des points acquis mais fragmentaires.

Les mécanismes suspectés, les plus vraisemblables actuellement, font appel au rôle d'un signal qui, reflet de l'état métabolique du sujet et agissant au niveau central - hypothalamique - , induirait des adaptations métaboliques et comportementales régulatrices.

Cette hypothèse générale a été proposée par quelques auteurs (Nicolaidis, 1980 ; Oomura, 1976 ; Panksepp, 1974) et des tentatives de vérifications expérimentales par d'autres. Ainsi, Friedman et Ramirez (1985) proposent l'idée selon laquelle constitution et mobilisation des réserves modifiant l'approvisionnement en 
métabolites utilisables affecteraient indirectement la prise alimentaire. Ils étudient le rat rendu hyperphagique et obèse par traitement insulinique. A la cessation du traitement, une augmentation transitoire de mobilisation des graisses est strictement parallèle à l'hypophagie en ce qui concerne aussi bien le décours temporel que la perte de poids qu'elle induit. II est par ailleurs bien établi que le facteur principal de l'augmentation de la cétogénèse est représenté par la lipolyse périphérique (Langhans et al., 1985). L'hypophagie qui survient pourrait donc être due à un changement du devenir des substrats lipidiques, non plus emmagasinés, mais oxydés et préférentiellement par la voie de la cétogénèse. Le rôle du foie et de son innervation afférante, comme intermédiaire dans le processus, n'est pas exclu.

Kasser et al. (1985, a, b) étudient in vitro le métabolisme du glucose et des acides gras au niveau du foie, du cortex cérébral, de l'hypothalamus latéral (HL) et ventromédian (HVM) ; ils comparent rats de poids normal et rats mis préalablement en souspoids et en surpoids. Ils montrent qu'une chute de poids imposée, entraînant I'hyperphagie à la cessation du traitement, se traduit au niveau du foie et du HL par une augmentation de $40 \%$ dans l'oxydation des acides gras et une baisse de $9 \%$ dans celle du glucose. Chez les rats mis en surpoids, et donc anorexiques, les auteurs observent une réduction de $36 \%$ dans l'oxydation du glucose. Les mêmes auteurs (1985), considérant les voies d'oxydation du glucose, montrent que, au niveau du $\mathrm{HL}$ le flux de glucose utilisant la voie du shunt de l'acide $\gamma$-amino-butyrique (GABA) est réduit chez les rats en souspoids et élevé chez les rats en surpoids. Au niveau du HVM, le flux du glucose empruntant la voie des pentoses est réduit chez les rats affamés, élevé chez les rats anorexiques.

Ces résultats montrent que, dans les zones cérébrales spécifiques (HL-HVM), le métabolisme du glucose et celui des acides gras répondent aux changements de l'état métabolique périphérique. De nombreuses données (revues par Kasser et al.) laissent penser que le métabolisme de ces zones hypothalamiques modulant leur activité, il s'ensuivrait une commande régulatrice de la prise alimentaire.

L'insulinémie basale des sujets humains ou animaux est fortement corrélée à leur adiposité. Cette relation demeure quand le poids et donc la masse des tissus adipeux sont expérimentalement manipulés (Woods et al., 1974). II est bien connu maintenant que le taux d'insuline dans le liquide céphalorachidien (LCR) est le reflet amorti, intégré, des variations de l'insulinémie (Owen et al., 1974 ; Stein et al., 1983 ; Woods et Porte, 1977).

Par ailleurs, il a été rapporté que certaines régions cérébrales, en particulier hypothalamiques, impliquées dans le contrôle métabolique sont particulièrement riches en insulinorécepteurs (Figlewiez et al., 1983 ; Van Houten et Posner, 1981). Enfin, des études récentes ont établi que l'augmentation expérimentale du niveau d'insuline dans le LCR (Brief et Davis, 1984 ; Woods et al., 1979) ou directement au niveau hypothalamique, induit une réduction de prise alimentaire ; l'injection in situ (HVM) d'anticorps antiinsuline l'augmente (Strubbe et Mein, 1977). Des injections périphériques d'insuline marquée (125 I) ont bien démontré chez le rat une liaison centrale et préférentielle au niveau de I'hypothalamus ventro-médian.

Ainsi, l'insulinémie périphérique, indicateur d'adiposité, indicateur de l'utilisa- 
tion des carburants, modulant le niveau d'insuline centrale pourrait, sur le jeu d'une liaison hypothalamique, réguler la prise alimentaire et donc l'adiposité. Cette hypothèse est séduisante ; cependant il faut remarquer que certaines études récentes ne la confirment pas. II faut des changements très importants de l'insulinémie périphérique pour faire varier, très faiblement d'ailleurs, le taux insulinique du liquide céphalorachidien. Les variations périphériques physiologiques ne semblent pas affecter l'insuline du liquide céphalorachidien (Manin et al., 1986). Par ailleurs, les relations entre insuline du tissu cérébral et celle du liquide céphalorachidien ne sont pas clairement élucidées.

Les trois groupes de travaux et suggestions qui viennent d'être résumés sont complémentaires. Ils donnent un aperçu réel de la direction actuelle des préoccupations dans ce domaine.

Les situations qui ont été évoquées et analysées montrent que la régulation du bilan d'énergie est précise et solide. II semble cependant qu'elle puisse être plus aisément mise en défaut lorsqu'interviennent des contingences hédoniques, autrement dit lorsque la composante « plaisir » de l'ingestion alimentaire est prise en compte.

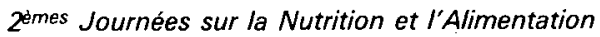
des Herbivores, I.N.R.A., Paris, 19 et 20 mars 1986.

\section{Références}

ADOLPH E. F., 1947. Urges to eat and drink in rats. Am. J. Physiol., 151, 110-125.

ASHWELL M., MEADE C. J., 1980. Fat transplantation in mice as a model for the study of concurrent fat loss and fat gain. Proc. Nutr. Soc., 39, A41.

ASTRUP A., BÜLOW J., CHRISTENSEN N. J., MADSEN J., QUAADE J., 1986. Facultative thermogenesis induced by carbohydrate : a skeletal muscle component mediated by epinephrine. Am. J. Physiol., 250, E 226-E 229.

BOGARDUS C., O'CONNEL M., JOHNSON M. J., HORTON E. S., SIMS E. A. H., DANFORTH E., 1982. Diet-induced alterations in total and free thyroid hormone concentrations during hypocaloric diets with and without carbohydrate. The amer. Thyroid Assoc. Quebec.

BOOTH D. A., 1972. Caloric compensation in rats with continuous or intermittent access to food. Physiol. Behav., 8, 891-892.

BOOTH D. A., 1972. Conditioned satiety in the rat. J. comp. Physiol. Psychol., 81, 457-471.

BOYLE P. C., STORLIEN L. H., KEESEY R. E., 1978. Increased efficiency of food utilization following weight loss. Physiol. Behav., 21, 261-264.

BRIEF D. J., DAVIS J. D., 1984. Reduction of food intake and body weight by chronic intraventricular insulin infusion. Brain Res. Bull., 12, 571-575.

BURGER G., BURGER M., WIMPFHREIMERT K., DANFORTH F., 1980. Interrelationships between energy metabolism and thyroid hormone metabolism during starvation in rat. Acta endocrinol., 93, 322-331.

CAMPBELL R. G., HASHIM S. A., VAN ITALLIE T. B., 1971. Studies of food intake regulation in man. Responses to variations in nutritive density in lean and obese subjects. New Engl. J. Med., 285, 1402-1407.

CASTONGUAY T. W., 1981. Dietary dilution and intake in the cat. Physiol. Behav., 27, 547-548.

COLLIER G. H., 1985. Satiety : an ecological perspective. Brain Res. Bull., 14, 693-700.

CUMMING M. C., MORRISON S. A., 1960. The total metabolism of rats during fasting and refeeding. J. Physiol., 154, 219-243.

DALVIT-Mc PHILLIPS S. P., 1983. The effect of the human menstrual cycle on nutrient intake. Physiol. Behav., 31, 209-212. 
DANFORTH E., HORTON E. S., SIMS E. A. H., 1979. Dietary-induced alterations in thyroid hormone metabolism during overnutrition. J. clin. Invest., 64, 1336-1347.

DANFORTH E., 1983. The role of thyroid hormones and insulin in the regulation of energy metabolism. Am. J. chin. Nutr., 38, 1006-1017.

DAUNCEY M. J., 1981. Influence of mild cold on $24 \mathrm{~h}$. energy expenditure, resting metabolism and diet-induced thermogenesis. Br. J. Nutr., 45, 257-267.

DONHOFFER S. Z., VONOTSKY J., 1947. The effect of thyroxine on food intake and selection. Am. J. Physiol., 150, 334-339.

FIGLEWICZ D. P., DORSA D., IKEDA H., STEIN L. J., BASKIN D., WOODS S. C., 1983. Brain insulin binding and content are altered by streptozotocin treatment in rats. Diabetes, 32 , 134A (abstract).

FRIEDMAN M. I., RAMIREZ I., 1985. Relationship of fat metabolism to food intake. Am. J. clin. Nutr., 42, 1093-1098.

GALBO H., 1983. Hormonal and metabolic adaptation to exercise. 1-116. 1st. ed. Thieme Stratton Inc., Stuttgart and New-York.

GALBO H., GOLLNICK P. D., 1984. Hormonal changes during and after exercise. Med. sport Sci., 17, 97-110.

GLEESON M., BROWN J. F., WARING J. J., STOCK M. J., 1979. Thermogenic effects of diet and exercise. Proc. Nutr. Soc., 38, A82.

GLEESON M., BROWN J. F., WARING J. J., STOCK M. J., 1982. The effects of physical exercise on metabolic rate and dietary-induced thermogenesis. Br. J. Nutr., 47, 173-183.

HANSEN B. C., JEN K. L. C., KAIBBS P., 1981. Regulation of food intake in monkey: response to caloric dilution. Physiol. Behav., 26, 479-486.

HILL J. O., LATIFF A., DI GIROLAMO M., 1985. Effects of variable caloric restriction on utilisation of ingested energy in rats. Am. J. Physiol., 248, R549-R559.

HSIEH A. C. L., CARLSON L. D., GRAY G., 1957. Role of the sympathetic nervous system in the control of chemical regulation of heat production. Am. J. Physiol., 190. 247-251.

JAMES D. E., BURLEIGH K. M., KRAEGEN E. W., CHISHOLM D. J., 1983. Effect of acute exercise and prolonged training on insulin response to intraveinous glucose in vivo in rats. J. appl. Physiol., 55. 1660-1664

JANSKY L., 1983. Humoral control of hypermetabolic and hypometabolic states. J. Physiol. (Paris), $78,872$.

KASSER T. R., HARRIS R. B. S., MARTIN R. J., 1985a. Level of satiety: fatty acid and glucose metabolism in three brain sites associated with feeding. Am. J. Physiol., 248, R447-R452.

KASSER T. R., HARRIS R. B. S., MARTIN R. J., 1985b. Level of satiety : GABA and pentose shunt activities in three brain sites associated with feeding. Am. J. Physiol., 248, R453-R458.

KATCH V. L., MARTIN R., MARTIN J., 1979. Effect of exercise intensity on food consumption in the male rats. Am. J. clin. Nutr., 32. 1401.

KRALY F. S., BLASS E. M., 1976. Increased feeding in rats at a low ambient temperature. In Hunger: basic mechanisms and clinical implications. Ed. D. NOVIN, W. WYRWICKA, G. BRAY. Raven Press New-York.

LANDSBERG L., YOUNG J. B., 1981. Diet-induced changes in sympatho-adrenal activity: implications for thermogenesis. Life Sci. 28. 1801-1819.

LANDSBERG L., SAVILLE M. E., YOUNG J. R., 1984. Sympathoadrenal system and regulation of thermogenesis. Am. J. Physiol., 247, E181-E189.

LANGHANS W., PANTEL K., SCHARRER E., 1985. Ketone kinetics and D-(-)-3-hydroxybutyrate induced inhibition of feeding in rats. Physiol. Behav., 34, 579-582.

LEBLANC J., JOBIN M., COTE J., SANSON P., LABRIE A., 1985. Enhanced metabolic response to caffeine in exercise trained human subjects. J. appl. Physiol., 59, 832-837.

LEBLANC J., NADEAU A., RICHARD D., TREMBLAY A., 1981. Studies on the sparing effect of exercise on insulin requirements in human subjects. Metabolism, 30, 1119-1125.

LEBLANC J., TREMBLAY A., RICHARD D., NADEAU A., 1983. Daily variations of plasma glucose and insulin in physically-trained and sedentary subjects. Metabolism, 32, 552-558. 
LE MAGNEN J., DEVOS M., 1980. Parameters of the meal pattern in rat : their assessment and physiological significance. Neurosci. Biobehav. Rev., 4, Suppl. 1, 1-11.

LESHNER A., COLLIER G., SQUIBB R. L., 1971. Dietary self-selection at cold temperatures. Physiol. Behav., 6, 1-3.

LESHNER A., SIEGEL H. I., COLLIER G., 1972. Dietary self-selection by pregnant and lactating rats. Physiol. Behav., 8, 151-154.

LEUNG P. M. B., HORWITZ B. A., 1976. Free-feeding patterns of rats in response to changes in environmental temperature. Am. J. Physiol., 213, 1220-1224.

LEVIN B. E., TRISCARI J., SULLIVAN A. C., 1983. Altered sympathetic activity during development of diet-induced obesity in the rat. Am. J. Physiol., 244, R347-R355.

LEWIS S. F., TAYLOR F. W., GRAHAM R. M., PETTINGER W. A., SCHUTTE J. E., BLOMOUIST C. G., 1983. Cardiovascular responses to exercise as function of absolute and relative workload. J. appl. Physiol., 54, 1314-1323.

LOUIS-SYLVESTRE J., 1984. Mécanismes de l'induction de l'hyperphagie et de l'obésité par le régime cafétéria: hypothèses. Cah. Nutr. Diet., XIX (4), 197-204.

MANIN M., MIZRAHI M., WYE P., BALAGE M., GRIZARD J., 1986. The influence of acute hyperinsulinemia on the insulin related material in brain, testis, liver and kidney (soumis).

MILLER D. S., MUMFORD P. G., 1967. Thermogenesis in overeating man. Am. J. clin. Nutr., 20, 1212-1222.

MONDON C. E., DOLKAS C. B., REAVEN G. M., 1980. Site of enhanced insulin sensitivity in exercise-trained rats at rest. Am. J. Physiol., 239, E169-E177.

MORTON J. H., ADDITON H., ADDITON R. G., HUNT L., SULLIVAN J. J., 1953. A clinical study of premenstrual tension. Am. J. Obstet. Gynecol, 65, 1182-1191.

NANCE D. M., BROMLEY B., BARNARD R. J., GORSKI R. A., 1977. Sexually dimorphic effects of forced exercise on food intake and body weight in the rat. Physiol. Behav., 19, 155-158.

NICOLAIDIS S., 1980. Hypothalamic convergence of external and internal stimulation leading to early ingestive and metabolic responses. Brain Res. Bull., 5, 97-101.

O'HARA W. J., ALLEN C., SHEPHARD J., ALLEN G., 1979. Fat loss in the cold. A controlled study. J. appl. Physiol., 46, 872-877.

OOMURA Y., 1976. Significance of glucose, insulin and free fatty acid on the hypothalamic feeding and satiety neurons, 145-157. In Hunger: basic mecanisms and clinical implications. Raven Press. New York.

OWEN O. E., REICHARD G. A., BODEN G., SHUMAN C. R., 1974. Comparative measurements of glucose, $\beta$-hydroxy-butyrate, acetoacetate and insulin in blood and cerebrospinal fluid during starvation. Metabolism. 23, 7-12.

PANKSEPP J., 1974. Hypothalamic regulation of energy balance and feeding behavior. Fed. Proc., 33. 1150-1165.

PERKINS M. N., ROTHWELL N. J., STOCK M. J., STONE T. W., 1981. Activation of brown adipose tissue thermogenesis by the ventromedial hypothalamus. Nature, 289, 401.

RAVUSSIN E., BOGARDUS C., SCHWARTZ R. S., ROBBINS D. C., WOLFE R. R., HORTON E. E., DANFORTH E., SIMS E. A. H., 1985. Glucose-induced thermogenesis and insulin resistance in man. Int. J. Obesity, 9, suppl. 2, 103-109.

RICHARD D., LEBLANC J., 1980. Effects of physical training and food restriction and glucose tolerance in male and female rats. Am. J. clin. Nutr., 33, 2588-2594.

ROLLS B. J., ROWE E. A., 1982. Pregnancy and lactation in the obese rat : effects on maternal and pup weights. Physiol. Behav., 28, 393-400.

ROLLS B. J., VAN DUIJVENVOORDE P. M., ROWE E. A., 1984. Effects of diet and obesity on bodv weight regulation during pregnancy in the rat. Physiol. Behav., 32, 161-168.

ROTHWELL N. J., SAVILLE M. E., STOCK M. J., 1985. Hormonal and metabolic responses to fasting and refeeding. Int. J. Obesity, 9, suppl. 2, 49-54.

ROTHWELL N. J., STOCK M. J., 1979. Regulation of energy balance in two models of reversible obesity in the rat. J. comp. Physiol. Psychol., 93, 1024-1034.

ROTHWELL N. J., STOCK M. J., 1979. A role for brown adipose tissue in diet-induced thermogenesis. Nature, 281, 31-35. 
ROTHWELL N. J., STOCK M. J., 1983. Effects of age on diet-induced thermogenesis and brown adipose tissue metabolism in the rat. Int. J. Obesity, 7, 583.

ROTHWELL N. J., STOCK M. J., 1984. Influence of alcohol and sucrose consumption on energy balance and brown fat activity in the rat. Metabolism, 33, 768-771.

ROWE J. W., YOUNG J. B., 1981. Effect of insulin and glucose infusions on sympathetic nervous system activity in normal man. Diabetes, 30, 219-225.

SCHLUSSER G. C., ORLANDO J., 1978. Fasting decreases triiodothyronine receptor capacity. Science, 199, 685-688.

SEGAL K. A., GUTIN B., 1983. Thermic effects of food and exercise in lean and obese women. Metabolism, 32, 581-590.

SEGAL K. R., GUTIN B., NYMAN A., PI-SUNYER F. X., 1985. Thermic effect of food at rest, during exercise, and after exercise, in lean and obese men of similar body weight. J. clin. Invest., 76, 1107-1112.

SNOWDON C. T., 1969. Motivation, regulation, and the control of meal parameters with oral and intragastric feeding. J. Comp. Physiol. Psychol., 69, 91-100.

STEIN L. J., DORSA D. M., BASKIN D. B., 1983. Immunoreactive insulin levels are elevated in the cerebrospinal fluid of genetically obese Zucker rats. Endocrinology, 113, 2299-2301.

STOCK M. J., ROTHWELL N. J., 1985. Factors influencing brown fat and the capacity for diet-induced thermogenesis. Int. J. Obesity, 9, suppl. 2, 9-15.

STOKHOLM K. H., HANSEN M. S., 1983. Lowering of serum total T3 during a conventional slimming regime. Int. J. Obesity, 7, 195-199.

STRUBBE J. H., MEIN C. G., 1977. Increased feeding in response to bilateral injections of insulin antibodies in the V.M.H. Physiol, Behav., 19, 309-313.

TRAYHURN A., 1985. Brown adipose tissue thermogenesis and the energetics of lactation in rodents. Int. J. Obesity, 9, suppl. 2, 81-88.

TREIT D., SPETCH M. L., 1986. Caloric regulation in the rat : control by two factors. Physiol. Behav., 36, 311-317.

VAN ES A. J. H., VOGT J. E., DEURENBERG P., VAN DER BECK E. J., 1982. Energy balance of humans at three levels of metabolisable energy intake. Proc. Nutr. Soc., 41, A34.

VAN HOUTEN M., POSNER B. I., 1981. Insulin receptors in the central nervous system : localisation and characteristics. Curr. Views Insul. Recep., 41, 75-91.

VINTEN J., PETERSEN L. N., SONNE B., GALBO H., 1985. Effect of physical training on glucose transporters in fat cell fractions. Biochim. Biophys. Acta, 841, 223-227.

WADE G. N., GRAY J. M., 1979. Gonadal effects on food intake and adiposity : a metabolic hypothesis. Physiol. Behav., 22, 583-593.

WADE G. N., ZUCKER I., 1970. Modulation of food intake and locomotor activity in female rats by diencephalic hormone implants. J. comp. Physiol. Psychol., 72, 328-336.

WARDZALA L. J., CRETTAZ M., MORTON E. D., JEANRENAUD B., MORTON E., 1982. Physical training of lean and genetically obese Zucker rats: effect on fat cell metabolism. Am. J. Physiol., 243, E418-E426.

WARNOLD I., LENNER R. A. L., 1977. Evaluation of the heart rate method to determine the daily energy expenditure in disease. A study in juvenile diabetics. Am. J. clin. Nutr., 30 , 304-315.

WOO R., PI-SUNYER F. X., 1985. Effect of increased physical activity on volontary intake in lean women. Metabolism, 34, 836-841.

WOODS S. C., DECKE E., VASSELLI J. A., 1974. Metabolic hormones and the regulation of weight. Psychol., Rev., 81. 26-43.

WOODS S. C., LOTTER E. C., Mc KAY L. D., PORTE D. Jr., 1979. Chronic intracerebroventricular infusion of insulin reduces food intake and body weight of baboons. Nature, 282, 503-505.

WOODS S. C., PORTE D., 1977. Relationship between plasma and cerebrospinal fluid insulin levels of dog. Am. J. Physiol., 233, E331-E334.

WURTMAN J. J., BAUM M. J., 1980. Estrogen reduces total food and carbohydrate intake, but not protein intake, in female rats. Physiol. Behav., 24, 823-827.

YOUNG J. B., LANDSBERG L., 1977. Suppression of sympatnetic nervous system during fasting. Science, 196, 1473-1475. 
YOUNG J. B., LANDSBERG L., 1977. Stimulation of sympathetic nervous system during sucrose feeding. Nature, 269, 615-617.

YOUNG J. B., NANCE D., GORSKI A., 1978. Dietary effects upon food and water intake and responsiveness to estrogen, 2-deoxy-glucose and glucose in female rats. Physiol. Behav., 21, 395-403.

ZAWALICH W., MATURO S., FELIG P., 1982. Influence of physical training on insulin release and glucose utilization by islet cells and liver glucokinase activity in the rat. $A m . J$. Physiol., 243, E464-E469. 